\title{
DIAGNÓSTICO DA HIPERTENSÃO ARTERIAL
}

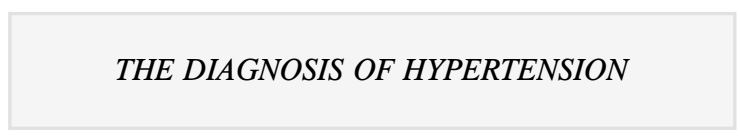

Décio Mion $\mathrm{Jr}^{1}$. , Angela Pierin², Sara Krasilcic ${ }^{3}$, Luis Celso Matavelli ${ }^{3}$, José Luiz Santello ${ }^{4}$

Docente $^{1,2}$. Departamento de Clínica Médica, Disciplina de Nefrologia ${ }^{1}$; Escola de Enfermagem²; Médico Pós-graduando³; Coordenador ${ }^{4}$ - Liga de Hipertensão Arterial - Hospital das Clínicas da Faculdade de Medicina da Universidade de São Paulo. CorrespondênCIA: Hospital das Clínicas da Faculdade de Medicina de São Paulo. Instituto Central - Laboratório de Hipertensão, $7^{\circ}$ andar. Avenida Dr. Eneas Carvalho de Aguiar, 44 São Paulo - SP - CEP: 05403-000

MION Jr D et al. Diagnóstico da hipertensão arterial. Medicina, Ribeirão Preto, 29: 193-198, abr./set., 1996.

RESUMO: O diagnóstico da hipertensão requer medida precisa da pressão arterial. Vários fatores relacionados ao observador, paciente, equipamento e à técnica podem alterar a medida da pressão arterial. Em relação ao observador, deve-se evitar preferências por dígitos, velocidade de deflação muito rápida e pressão excessiva sobre a artéria braquial; os olhos do observador devem ser mantidos ao nível da coluna de mercúrio. No paciente, deve-se excluir ansiedade, distensão vesical, exercício físico, dor, fumo ou alimentação recente. Problemas nos estigmomanômetros e relação inadequada entre a largura do manuguito e o braço do paciente, também, podem causar erros na medida da pressão arterial.

UNITERMOS: Hipertensão; diagnóstico. Esfigmomanômetros.

\section{DIAGNÓSTICO DA HIPERTENSÃO ARTERIAL}

O diagnóstico de hipertensão arterial é um ato médico que, baseado num procedimento relativamente simples, a medida da pressão arterial, envolve a grande responsabilidade de decidir se um paciente é normotenso ou hipertenso. As conseqüências de um diagnóstico errôneo são desastrosas. O diagnóstico de normotensão, num hipertenso, irá privá-lo dos benefícios do tratamento, ao passo que o de hipertensão, num normotenso, irá submetê-lo aos malefícios do tratamento desnecessário.

O diagnóstico em hipertensão arterial é baseado na anamnese, exame físico e exames complementares que auxiliam na realização do diagnóstico da doença propriamente dita, sua etiologia, grau de comprometimento de órgãos-alvo e na identificação dos fatores de risco cardiovascular associados.
O II Consenso Brasileiro de Hipertensão Arterial $^{1,2}$ recomenda que a história clínica e exame físico do paciente hipertenso sigam as orientações clássicas destes procedimentos, mas sejam orientados para identificar:

\section{Anamnese:}

1) sexo, idade, raça e condição sócio-econômica;

2) duração da hipertensão;

3) tratamento prévio, adesão e reações adversas;

4) sintomas sugestivos de isquemia cerebral, miocárdica e de membros inferiores; dispnéia, edema e perda de visão;

5) sintomas sugestivos de hipertensão secundária (Tabela I);

6) história familiar de hipertensão, acidente vascular encefálico, infarto do miocárdio, doença renal, diabete, dislipidemia, morte prematura e súbita; 
7) tensão emocional, consumo de sal e bebidas alcoólicas;

8) fatores de risco associados: dislipidemias, tabagismo, diabete, obesidade, e sedentarismo;

9) medicamentos que possam elevar a pressão arterial ou interferir no seu tratamento. A apnéia do sono, também, deve ser investigada quando há roncos durante o sono e sonolência diurna.

Tabela I: Dados sugestivos de hipertensão arterial secundária

1. Facies ou biotipo de doença que cursa com hipertensão: doença renal, hipertireoidismo, acromegalia, Cushing

2. Início da hipertensão antes dos 30 ou após os 50 anos

3. Diminuição de amplitude ou retardo da pulsação femural

4. Hipertensão grave e/ou resistente à terapia.

5. Presença de massas ou sopros abdominais

6. Tríade do feocromocitoma: palpitações, sudorese e cefaléia em crises

7. Aumento da creatinina sérica

8. Hipopotassemia espontânea (<3 meq/l)

9. Exame de urina anormal
6) exame de abdome; massas abdominais indicativas de rins policísticos, hidronefrose, tumores, aneurismas e sopros abdominais (aorta e artérias renais);

7) exame neurológico; comprometimento vascular encefálico;

8) exame de fundo de olho: identificar estreitamento arteriolar, cruzamentos artériovenosos patológicos, hemorragias, exsudatos e papiledema.

\section{Exames Laboratoriais}

Para a avaliação laboratorial mínima do hipertenso o II Consenso Brasileiro de Hipertensão Arterial recomenda:

1) exame de urina para pesquisa de elementos anormais e sedimento;

2) dosagens de creatinina e potássio séricos;

3) glicemia de jejum;

4) colesterol total para homens acima de 20 anos e mulheres na menopausa. Quando o colesterol total exceder $200 \mathrm{mg} \%$, recomenda-se a dosagem das demais frações lipídicas.

5) eletrocardiograma.

Os pacientes que apresentem alterações clínicas ou laboratoriais sugestivas de hipertensão secundária devem ser investigados, através de métodos específicos, preferencialmente em centros especializados de referência.

\section{Medida da Pressão Arterial}

Na prática clínica, o diagnóstico da hipertensão arterial é realizado através da medida indireta da pressão arterial, empregando esfigmomanômetro e técnica auscultatória com estetoscópio ${ }^{3,4}$. É importante destacar que a monitorização ambulatorial da pressão arterial é um método auxiliar útil em hipertensão, mas não está indicada no diagnóstico de rotina da hipertensão arterial.

A medida da pressão é procedimento simples, porém sujeito a vários fatores de erro. Assim, para medida precisa da pressão arterial e, conseqüentemente, diagnóstico correto da hipertensão, é muito importante adotar os cuidados e critérios, descritos abaixo, relacionados ao observador, paciente, ambiente, equipamento e técnica. 


\subsection{Observador}

O observador que efetua a medida da pressão arterial deve estar treinado para a realização do procedimento a fim de evitar:

a) preferência por valores de pressão arterial terminados com dígitos zero ou cinco. A leitura dos valores deve refletir, precisamente, a escala do manômetro que possui números terminados pelos dígitos zero, dois, quatro, seis, oito e dez;

b) leitura errônea dos valores da escala, devido à posição incorreta dos olhos. Os olhos do observador devem estar posicionados ao nível do topo da coluna de mercúrio ou incidir, diretamente, sobre o mostrador do manômetro aneróide para permitir correlação exata entre o som auscultado e o valor correspondente na escala graduada.

c) pressão excessiva sobre o diafragma do estetoscópio que pode deformar a artéria braquial e ocasionar alteração do som auscultado.

d) inflação excessiva da bolsa de borracha que pode causar dor e levar a leituras falsamente elevadas:

e) deflação muito rápida que pode ocasionar leitura, falsamente, baixa para a pressão sistólica e, falsamente, elevada para diastólica; e

f) mãos e equipamentos excessivamente frios que podem levar a alteração da pressão arterial pela reação ao frio.

A interação entre o observador e o paciente, também, é fonte importante de interferência na medida da pressão arterial. Em nosso meio, Pierin, $1992^{5}$ comparou os valores de pressão arterial obtidos pelo médico, enfermeira e paciente/familiar com os registrados pela monitorização ambulatorial da pressão arterial e verificou que os obtidos pela enfermeira e paciente/familiar foram os que mais se aproximaram dos registrados na monitorização, método que não sofre influência do observador. Muitas vezes, a interação entre o médico e o paciente pode levar ao que se denomina fenômeno ou hipertensão do "avental branco". Nesta condição, a pressão verificada pelo médico é mais elevada do que a obtida por outro profissional ou a registrada pela monitorização ambulatorial da pressão arterial. Ocorre o fenômeno do "avental branco" quando verifica-se elevação da pressão sem alteração no diagnóstico de normotensão ou hipertensão e hipertensão do "avental branco" quando o paciente passa da situação de normotensão para hipertensão, quando a pressão é medida pelo médico.
A hipertensão do "avental branco" ocorre em cerca de $20 \%$ dos hipertensos leves.

Portanto, é fundamental evitar tensão e ansiedade do paciente, estabelecendo uma relação de confiança por meio de esclarecimentos sobre o procedimento de medida da pressão arterial e sua finalidade.

\subsection{Ambiente}

O ambiente onde se realiza a medida da pressão deve ser calmo e ter temperatura agradável. Quando a medida é realizada em locais, como Pronto-Socorro ou em campanhas em "Shopping Centers", é preferível alertar o paciente para que confirme a medida em outra situação do que firmar o diagnóstico, exceto em situações de emergência hipertensiva.

\subsection{Paciente}

O paciente deve permanecer em repouso 5 a 10 minutos, antes da medida da pressão, depois de ter esvaziado a bexiga. Deve-se evitar atividade física, alimentação, fumo, ingestão de bebida alcoólica ou café pelo menos 30 minutos antes da medida. É importante indagar o paciente sobre ingestão de drogas que interferem com os mecanismos de regulação da pressão.

A posição recomendada para medida da pressão é a sentada, com o tronco recostado e relaxado no encosto da cadeira, as pernas relaxadas sem cruzar, com o braço em que o manguito será colocado ao nível do coração, livre de roupas, a palma da mão voltada para cima e o cotovelo ligeiramente fletido. A medida da pressão na posição ereta é útil para identificação de hipotensão postural.

\subsection{Equipamento}

O esfigmomanômetro aneróide ou de coluna de mercúrio são os aparelhos empregados para a medida da pressão arterial. É fundamental que estejam, devidamente, calibrados para que a medida seja correta. A verificação da calibração deve ser realizada, pelo menos, a cada 6 meses.

A verificação da calibração do aparelho de coluna de mercúrio é efetuada através da inspeção visual do nível de mercúrio, em relação à escala da coluna de vidro, estando o manguito desinsuflado. Quando o nível de mercúrio coincide com o valor zero da escala o aparelho está calibrado. Quando a borda do menisco da coluna de mercúrio estiver abaixo ou acima do valor zero há necessidade de colocar ou retirar mercúrio do reservatório. 
A verificação da calibração do manômetro aneróide exige a realização do teste de calibração contra aparelho de coluna de mercúrio. Neste teste, emprega-se conector em forma de "Y", ligado numa extremidade ao tubo de borracha do aparelho de coluna de mercúrio, na outra extremidade ao tubo de borracha do manômetro aneróide a ser testado e na terceira extremidade à pêra de borracha que será utilizada para inflar o sistema. O sistema deve ser inflado até ultrapassar $250 \mathrm{mmHg}$ da escala de vidro do aparelho de coluna de mercúrio e ao se proceder a deflação, verifica-se a correspondência de valores na escala dos dois manômetros de $50 \mathrm{em} 50 \mathrm{mmHg}$. Caso a diferença entre as duas escalas seja $\pm 3 \mathrm{mmHg}(1 \%$ da escala total) em qualquer um dos valores, considera-se o aparelho descalibrado.

Esta verificação da calibração tem sido negligenciada pelos médicos. Mion \& Pierin (dados não publicados) avaliaram 524 esfigmomanômetros (61\% de coluna de mercurio e $39 \%$ aneróide) e verificaram que 59\% dos aneróides e 35\% dos aparelhos de coluna de mercúrio estavam descalibrados, evidenciando que a verificação da calibração e manutenção dos aparelhos não está sendo realizada com a frequiência necessária.

Além dos cuidados com a calibração, a coluna de mercúrio deve ser inspecionada, periodicamente, para identificação de sinais de oxidação e sujidade. A obstrução do filtro do topo da coluna pode levar a leituras incorretas, devido à dificuldade de elevação durante a inflação do sistema e pode ocasionar oscilação excessiva quando do fechamento da válvula, requerendo limpeza ou troca periódica.

Outro aspecto importante em relação ao equipamento é o manguito porque a largura da bolsa inflável do manguito, também, pode ser fonte de erro na medida da pressão arterial. Assim, quando a bolsa inflável é estreita em relação à circunferência do braço do paciente, a leitura de pressão pode ser falsamente elevada, levando ao diagnóstico errôneo de hipertensão arterial. Por outro lado, quando a bolsa é larga, a leitura pode ser falsamente baixa, levando ao diagnóstico errôneo de normotensão. A relação recomendada entre a largura da bolsa e a circunferência do braço do paciente é de 0,4. Assim, a largura da bolsa inflável deve ser $40 \%$ da circunferência do braço, ou seja, a relação entre a largura da bolsa inflável e a circunferência do braço do paciente deve ser 1:2. Além disso, o seu comprimento deve corresponder a
$40 \%$ da circunferência do braço e o seu comprimento deve envolver, pelo menos, $80 \%$ do braço.

O procedimento ideal para evitar este tipo de erro é o uso de manguito adequado à circunferência do braço de cada paciente ${ }^{6}$. No entanto, este método exige que o médico, antes da medida da pressão, meça a circunferência do braço do paciente com fita métrica, consulte a tabela para identificar o tipo de manguito adequado e instale o manguito selecionado no aparelho de pressão. Além de não ser prático, este método é oneroso porque o médico deveria possuir manguitos de vários tamanhos, pois o manguito padrão disponível no comércio que possui $12 \mathrm{~cm}$ de largura estaria indicado, somente, para braços com circunferência de $30 \mathrm{~cm}$.

Outro procedimento, mais simples e prático, empregado para minimizar este tipo de erro é corrigir a leitura obtida com manguito padrão, segundo as tabelas de correção disponíveis. Para facilitar ainda mais este procedimento Mion e cols $1986^{7}$ desenvolveram fita de correção da pressão que contém, numa escala superior, os valores de correção a serem somados ou subtraídos da leitura obtida para pressão sistólica e numa escala inferior para diastólica. Assim, ao se colocar o manguito no braço do paciente são identificados os valores a serem somados ou subtraídos da leitura obtida da pressão sistólica e diastólica. Esta fita de correção da pressão é apresentada em forma de fita adesiva para ser utilizada com manguito padrão com bolsa de $12 \mathrm{~cm}$ de largura e $23 \mathrm{~cm}$ de comprimento.

O sistema de válvula e tubos de borracha, também, necessitam "checagem" periódica para identificar envelhecimento da borracha, vazamentos nas conexões ou furos nas extensões. Este sistema deve possibilitar inflação rápida e deflação gradual.

Os aparelhos automáticos podem apresentar imprecisões mais facilmente que os aneróides e os de coluna de mercúrio e o seu uso rotineiro não é indicado para profissionais da área da saúde. Esses aparelhos podem ser úteis para os pacientes hipertensos controlarem sua pressão em casa.

\subsection{Procedimento da Medida da Pressão Arterial}

O paciente deve ser instruído previamente sobre tal procedimento, explicando as suas etapas e objetivos. É importante indagar se o paciente está com a bexiga vazia, se evitou atividade física, alimentação, fumo, ingestão de bebida alcoólica ou café, pelo 
menos 30 minutos antes da medida, e se ingeriu drogas que interferem com os mecanismos de regulação da pressão.

O procedimento de medida envolve as seguintes etapas:

a) deixar o paciente em repouso 5 a 10 minutos, em ambiente calmo, com temperatura agradável;

b) localizar pela palpação a artéria braquial no braço do paciente;

c) posicionar o manguito firmemente, evitando folgas e centralizando a bolsa de borracha sobre a artéria braquial, cerca de 2 a $3 \mathrm{~cm}$ acima da fossa antecubital. Nas posições sentada e ereta, manter o braço na altura do quarto espaço intercostal;

d) palpar o pulso radial e inflar o manguito até o desaparecimento deste pulso para identificação da pressão sistólica e desinflar, rapidamente, aguardando 15 a 30 segundos antes de inflar novamente;

e) posicionar o estetoscópio, suavemente, na fossa antecubital sobre a artéria braquial, evitando compressão excessiva. Deve-se empregar a campânula do estetoscópio, pois os sons auscultados são de baixa frequiência. Colocar o estetoscópio nos ouvidos com a curvatura voltada para frente.

f) inflar rapidamente, de $10 \mathrm{em} 10 \mathrm{~mm} \mathrm{Hg}$, até ultrapassar 20 a 30 mm Hg o nível da pressão sistólica e proceder a deflação em velocidade constante de 2 a $3 \mathrm{~mm} \mathrm{Hg}$, por segundo, que pode ser elevada para 5 a $6 \mathrm{~mm} \mathrm{Hg}$ por segundo, após a determinação da sistólica para evitar congestão venosa e desconforto para o paciente;

g) identificar a pressão sistólica no momento do aparecimento do primeiro som da série de sons (fase I de Korotkoff), que se intensifica com o aumento da velocidade de deflação e a pressão distólica quando desaparecem os sons (fase $\mathrm{V}$ de Korotkoff), auscultando cerca de 20 a $30 \mathrm{~mm} \mathrm{Hg}$ abaixo do último som para confirmar seu desaparecimento, e depois, proceder a deflação rápida e completa;

h) registrar os valores de pressão sistólica e diastólica, assim como a posição do paciente, braço em que foi medida a pressão e tamanho do manguito;

i) aguardar 1 a 2 minutos para realizar nova medida.

A técnica de medida de pressão arterial, também, pode constituir-se em uma fonte de erro. Em algumas situações, após a ausculta dos sons iniciais, estes desaparecem para reaparecerem em níveis mais baixos, o que provoca um vazio, chamado hiato auscultatório, que ocorre no final da fase I e início da fase II dos sons de Korotkoff. Este hiato pode prolongar-se até por $40 \mathrm{mmHg}$, levando à hipoestimação da pressão sistólica ou hiperestimação da diastólica. Para evitar este tipo de erro é que existe a orientação de palpar o pulso radial e inflar o manguito até o seu desaparecimento, garantindo que o nível da sistólica foi ultrapassado.

Quando houver dificuldade na ausculta dos sons, pode-se tentar intensificá-los, inflando o manguito após o braço do paciente estar elevado por 30 segundos, antes de colocar o braço na posição correta para medir a pressão arterial.

Em alguns pacientes, não há o desaparecimento do som, auscultando-se até o ponto zero. Nesta situação, que pode ocorrer em crianças e pacientes com insuficiência valvar aórtica, deve-se empregar a fase IV, que corresponde ao abafamento acentuado do som, para a caracterização da pressão diastólica, anotando-se os valores da sistólica/diastólica/zero mmHg.

MION Jr D et al. The diagnosis of hypertension. Medicina, Ribeirão Preto, 29: 193-198, apr./sept., 1996.

ABSTRACT: The diagnosis of hypertension requires accurate measurement of blood pressure. Blood pressure readings are altered by several factors related to the observer, patient, equipment and technique used. The observer must avoid digt preference, deflation rate too fast, heavy pressure on brachial artery and keep the eye level at the top of the column of mercury. The patient should shun anxiety, distention of urinary bladder, exertion, pain and recent smoking or food intake. Errors in blood pressure readings can also be caused by defects of sphigmomanometers and cuff size/arm size disparity.

UNITERMS: Hypertension; diagnosis. Sphigmomanometers. 


\section{REFERÊNCIAS BIBLIOGRÁFICAS}

1 - II CONSELHO BRASILEIRO DE HIPERTENSÃO ARTERIAL. J Bras Nefrol 16 (supl 2): S257-278, 1994.

2 - THE FIFTH REPORT OF THE JOINT NATIONAL COMMITTEE ON DETECTION, EVALUATION, AND TREATMENT OF HIGH BLOOD PRESSURE. Arch Inter Med 153: 154-183, 1993.

3 - RECOMENDATIONS FOR HUMAN BLOOD PRESSURE BY SPHYGMOMANOMETERS, ed. American Heart Association Washington, p1-34, 1987.

4 - PIERIN AMG \& MION Jr D. Como evitar erros na medição da pressão arterial. Cons Médico 2: 10-12, 1994.
5 - PIERIN AMG. Medidas da pressão arterial no ambulatório pelo cliente, enfermeira e médico comparadas a registros domiciliares. Tese de Doutorado, Escola de Enfermagem da USP, São Paulo, 119 p., 1992.

6 - ARCURI EAM. Estudo comparativo da medida indireta da pressão arterial com manguito de largura correta e com manguito de largura padrão. Arq Bras Cardiol 47: 143-144, 1986.

7 - MION JR D; SILVA H \& MARCONDES M. Device to correct the reading of blood pressure according to the patientís arm circumference. J Hypertens 4 (supl 15): S581, 1986.

Recebido para publicação em 16/01/96

Aprovado para publicação em 25/04/96 